\title{
CONSUMO PROBLEMÁTICO DE DROGAS Y TERAPIA OCUPACIONAL: COMPONENTES OCUPACIONALES EVALUADOS DURANTE EL PROCESO DE TRATAMIENTOY REHABILITACIÓN
}

\author{
PROBLEMATIC DRUG ABUSE AND OCCUPATIONAL THERAPY: \\ OCCUPATIONAL COMPONENTS ASSESSED DURING THE \\ PROCESS OF TREATMENT AND REHABILITATION
}

\section{Lisette Farias V. ${ }^{1}$, Verónica Guerra I. ${ }^{2}$, Tamara Cifuentes A. ${ }^{3}$ y Susana Rozas C. ${ }^{4}$ Profesora Guía: $M^{a}$ Elena Riveros E. ${ }^{5}$}

\section{Resumen}

El tema de esta investigación se enmarca dentro del área del Consumo problemático de Drogas y Terapia Ocupacional. El propósito de este estudio cualitativo, es explorar los Componentes Ocupacionales que terapeutas ocupacionales evalúan durante el proceso de tratamiento y rehabilitación del consumo problemático de drogas. Además, se exploran los modelos y evaluaciones utilizados durante este proceso. La selección del colectivo de estudio se realizó consultando el listado de Centros de Tratamiento y Rehabilitación obtenido de la base de datos del Consejo Nacional para el Control de Estupefacientes (CONACE).

1 Terapeuta Ocupacional, Lic. en Cs. de la Ocupación Humana, Universidad de Chile. Estudiante European Master of Science in Occupational Therapy. Dirección postal: Orvar Odds Väg 51, 11254 Estocolmo, Suecia. Fono Chileno: 5709486 Mail: lissette.farias@gmail.com

2 Terapeuta Ocupacional, Lic. en Cs. de la Ocupación Humana, Universidad de Chile. Diplomado en Gerontología Comunitaria, Universidad de Santiago de Chile. Proyecto de Integración Escolar, Corporación de Educación de Maipú. Dirección Postal: Av. San Pablo 3900, depto. 807, Quinta Normal. Fono: 06-8468818. Mail: vg.guerra.i@gmail.com

3 Terapeuta Ocupacional, Lic. en Cs. de la Ocupación Humana, Universidad de Chile. Programa de Tratamiento y Rehabilitación de personas con trastorno psiquiátrico severo. COSAM de Maipú. Dirección Postal: Vergara 564 depto. 301, Santiago Centro. Fono: 02-5346897. Mail: to.tamy@gmail.com

4 Terapeuta Ocupacional, Lic. en Cs. de la Ocupación Humana, Universidad de Chile. Centro de Rehabilitación Integral para niños y adolescentes Manantial. Dirección Postal: Los Leones 1480, Providencia. Fono: 08-1910791 Mail: susanarozas@ gmail.com

5 Terapeuta Ocupacional, Lic. en Cs. de la Ocupación Humana, Magíster en Salud Pública, Candidata Doctor en Salud Pública, Universidad de Chile. Jefa Área Internacional Comisión Nacional de Control de Estupefacientes-CONACE. Académica Universidad de Chile. Dirección Postal: Agustinas 1235 Piso 9, 8340422. Fono: 5100858. Mail: mriveros@conace.gov.cl 
La recolección de datos se llevó a cabo a través de la revisión de la literatura existente sobre el tema y una entrevista semiestructurada a siete Terapeutas Ocupacionales, con experiencia en el área de estudio. La entrevista se centró en los siguientes temas: Componentes Ocupacionales evaluados por terapeutas ocupacionales durante el proceso de Tratamiento y Rehabilitación, modelos y evaluaciones utilizadas por terapeutas ocupacionales y Componentes Ocupacionales que muestran mayor déficit.

Los resultados de esta investigación revelaron que la mayoría de los terapeutas ocupacionales evalúan los componentes ocupacionales de rutina/hábitos, roles, volición, tiempo libre e historia laboral en la etapa de ingreso a Centros de Tratamiento y Rehabilitación. Los resultados también revelaron una carencia de modelos y evaluaciones adecuadas y específicas desde la Terapia Ocupacional en el tema de estudio. Este estudio concluye en que existe una carencia de homogeneidad en el lenguaje, instrumentos de evaluación y los modelos utilizados entre Terapeutas Ocupacionales en los distintos Centros de Tratamiento y Rehabilitación.

\section{Palabras claves}

Componentes Ocupacionales, Evaluaciones e instrumentos desde la Terapia Ocupacional y Modelo de Ocupación Humana.

\section{Abstract}

This research topic is framed within the field of occupational therapy and problematic drug abuse. The purpose of this qualitative study is to explore what occupational components occupational therapists assess in the process of treatment and rehabilitation of problematic drug abuse. The study also explores the models and assessments used during this process.

The participants were selected out of the list of Treatment and Rehabilitation Centers, that provides Occupational Therapy intervention in an Intensive Outpatient Program. This list was obtained from the database of the National Council for Narcotics Control (CONACE).

Data was collected through literature review on the topic and semi-structured interviews with seven Occupational Therapists with experience in the field of Drug Addiction Treatment and Rehabilitation. The interviews focused on the following topics: occupational components that are assessed for occupational therapists during the process of Treatment and Rehabilitation, models or theoretical frameworks and assessments used during this process and occupational components most affected.

The results revealed that the majority of occupational therapists evaluate the occupational components of routine / habits, roles, volition, leisure and work history at the stage of admission to Treatment and Rehabilitation Centers. In addition, the results reveal a lack of appropriate and specific models and assessments from the occupational therapy perspective within the field of drug abuse. This study concludes that there is a lack of homogeneity in language, assessment tools and models used between the Occupational Therapists working in the Treatment and Rehabilitation Centers.

\section{Keywords}

Occupational Components, Assessments from Occupational Therapy perspective and Model of Human Occupation. 


\section{INTRODUCCIÓN}

El consumo problemático de drogas, es decir, "el consumo de larga duración o habitual de opiáceos, cocaína o anfetaminas" (1), representa uno de los problemas más significativos que enfrentan los países desarrollados alrededor del todo el mundo, con un impacto creciente en países como Chile. Esta epidemia representa un alto costo económico, tanto por la inversión económica y humana utilizada en su prevención y rehabilitación, como por los insuficientes resultados de ésta. El elevado número de muertes prematuras que conlleva, lo convierten en un tema trascendental en la administración de políticas de salud pública que enfatizan en prevención y rehabilitación.

Las estadísticas de los últimos años $(2,3,4)$, indican que la prevalencia del consumo de drogas, tanto lícitas como ilícitas, han aumentado o se han mantenido en nuestro país, según el tipo de droga. En general, los resultados han mostrado que las tasas más altas de consumo se sitúan en la Región Metropolitana. En relación a género y nivel socioeconómico, los hombres y el nivel socioeconómico bajo, siguen liderando las prevalencias de consumo. La población con mayor consumo se sitúa en el grupo de jóvenes entre 19 y 25 años, con una tendencia al aumento del consumo en el grupo de jóvenes entre los 26 y 34 años, los cuales forman parte del grupo motor productivo de la sociedad chilena.

De los resultados del estudio de la descripción del Perfil Ocupacional del Consumidor de Drogas (5), se deduce una pérdida de los hábitos, rutinas y roles en el consumidor problemático de drogas lo cual conlleva a una Disfunción Ocupacional que afecta al usuario en su contexto, sus relaciones y desenvolvimiento, limitando su actividad ocupacional e impactando su estado de salud.

De acuerdo a la revisión de la literatura existente en el tema y de los instrumentos de evaluación propios de la Terapia Ocupacional, se evidenció una ausencia de instrumentos específicos destinados a determinar los
Componentes Ocupacionales en el área del consumo problemático de drogas. Por lo tanto, el determinar estos componentes Ocupacionales, sería un aporte al quehacer de la Terapia Ocupacional en la realización de planes de intervención acorde a las necesidades y al perfil particular de éstos usuarios, además de contribuir en la investigación de la Terapia Ocupacional en ésta área.

Una investigación realizada en el año 2005 (6), arrojó dentro de sus resultados, una heterogeneidad en los enfoques de evaluaciones e intervención, llevados a cabo en los Programas de Rehabilitación de Drogodependencia en la Región Metropolitana. Los resultados de ésta investigación señalaron que las intervenciones de Terapia Ocupacional eran basadas en modificaciones de evaluaciones ya establecidas, ajustándolas a los tiempos disponibles tanto del establecimiento como de los usuarios y utilizando diversos marcos teóricos según las distintas características dadas por el contexto institucional y beneficiarios.

Otras investigaciones $(6,7,8)$ revelan que cada Terapeuta Ocupacional elige y adapta los instrumentos de evaluación según su criterio profesional, tiempo y factores institucionales que influencian el ejercicio de su profesión, lo cual puede provocar altos costos en tiempo y efectividad al realizar una evaluación del consumidor problemático de drogas sin instrumentos específicos para ésta área. Encontrándonos, finalmente con una falta de protocolos de intervención estandarizados y válidos para la Terapia Ocupacional en el área del Consumo Problemático de Drogas.

La Terapia Ocupacional tiene un amplio campo de posibles intervenciones en ésta área, tratando de restablecer, modificar o crear un nuevo sentido y significado a las ocupaciones de los usuarios con consumo problemático de drogas, generando una rutina diaria satisfactoria y funcional. A través de la ocupación, podemos identificar los intereses del sujeto, el nivel de satisfacción con su vida, los contextos donde se desempeña, las actividades a las que le otorga 
mayor importancia o a las que le dedica mayor tiempo y el grado de identificación que el sujeto posee con los roles y expectativas esperadas socialmente, entre otros.

El propósito de este estudio cualitativo, es explorar los Componentes Ocupacionales evaluados por terapeutas ocupacionales durante el proceso de tratamiento y rehabilitación del consumo problemático de drogas. Se espera que esta investigación, además permita explorar los modelos e instrumentos de evaluación más utilizados por los Terapeutas Ocupacionales en los distintos centros de tratamiento y rehabilitación, con el fin de aportar una base para la creación de un lenguaje consensuado en ésta área y un primer paso hacia la construcción de evaluaciones desde la Terapia Ocupacional específicas para el ámbito del consumo problemático de drogas.

\section{Pregunta de Investigación}

¿Cuáles son los Componentes Ocupacionales a evaluar en el proceso de Tratamiento y Rehabilitación del Consumo Problemático de Drogas?

\section{Supuestos}

- Existe una Disfunción Ocupacional en personas con consumo problemático de drogas $(5,9)$.

- La Terapia Ocupacional es la única disciplina que aborda la perspectiva de la Ocupación Humana, considerada central en la Rehabilitación y reinserción social de personas con problemas de consumo de drogas (8).

- La Terapia Ocupacional podría incidir en la efectividad del tratamiento y rehabilitación a través de una apropiada entrega de un repertorio de actividades que la persona puede desarrollar durante su tratamiento. Esta selección le ayuda al individuo a permanecer saludable y resistir el stress (10).

\section{Marco Conceptual}

\section{Definiciones y conceptos básicos}

a) Componentes Ocupacionales: Debido a la heterogeneidad en el leguaje conceptual utilizado por los participantes en este estudio, el grupo de investigadores decidió formular un concepto único y básico para reunir la información recolectada a través de las entrevistas. Éste concepto es denominado "Componentes Ocupacionales" y reúne las áreas ocupacionales, los patrones y habilidades de desempeño y aspectos del entorno definidos en el Marco de Trabajo para la Terapia Ocupacional: Dominio y Proceso (11).

b) Tratamiento y Rehabilitación en Drogadicción: Según el Comité de Expertos de la Organización Mundial de la Salud en Farmacodependencia, el término "tratamiento" se aplica al proceso que comienza cuando los usuarios de sustancias psicoactivas entran en contacto con un proveedor de servicios de salud o de otro servicio comunitario y puede continuar a través de una sucesión de intervenciones concretas hasta que se alcanza el nivel de salud y bienestar más alto posible (12).

c) Abuso de sustancias: Es uno de los trastornos por consumo de sustancias, que consiste en un patrón desadaptativo de consumo de sustancias que conlleva un deterioro o malestar clínicamente significativos, expresado por uno (o más) de los ítems clasificados en el Manual Diagnóstico y Estadístico de Trastornos Mentales de la Asociación Americana de Psiquiatría, DSM-IV durante un período de 12 meses (13).

\section{Modelo Teórico}

El Modelo de Ocupación Humana (14) organiza los conceptos de Ocupación en un marco de referencia basado en la Teoría General de Sistemas. El Modelo describe al hombre como un sistema abierto y dinámico, siendo el comportamiento ocupacional su output. Establece los aspectos de motivación, comportamiento, organización y aquellos relevantes para el entendimiento de la 
Ocupación. De acuerdo a este Modelo, toda Ocupación nace de una tendencia innata y espontánea del sistema humano, que lo lleva a explorar y a sentirse eficaz en el ambiente.

Este modelo propone que las elecciones para involucrarnos en cualquier comportamiento ocupacional, están influenciadas por disposiciones simbólicas y Autoconocimiento, las que se refieren a orientaciones emocionales y cognitivas hacia las ocupaciones, impactadas tanto por la experiencia previa como por anticipaciones hacia el futuro. Cada vez que se realiza una selección de actividad, se anticipa una experiencia o resultado particular y/o algún objetivo que se espera alcanzar. En el caso de las personas con consumo problemático de drogas, existe generalmente una alteración en el Autoconocimiento (5), lo que conlleva a una elección errónea de las ocupaciones, las cuales pueden encontrarse por sobre o bajo sus habilidades, situación que puede condicionar frustración o bajo nivel de satisfacción en la actividad. De ésta forma, la persona buscará actividades que le generen expectativas positivas y que indiscutiblemente lo lleven a alcanzar su objetivo. Dentro de éstas actividades se encuentran las relacionadas directa o indirectamente al consumo, el cual le provee de experiencias satisfactorias.

A su vez, el proceso de interpretar la experiencia genera un Autoconocimiento o percepción de sí mismo como actor en el mundo. Ésta conciencia permite construir complejas ideas acerca del futuro y sus posibilidades, por lo tanto contribuye a un proceso de selección ocupacional. Así, en el caso del consumo problemático de drogas, se configura un círculo vicioso, ya que a través del consumo y las actividades involucradas en él, la persona se "autoconoce" y se va reafirmando en dicha actividad, limitando sus proyecciones y posibilidades.

Por otro lado, la cultura provee un medio para entender y sentir acerca de la vida diaria. Las personas se comportan de una determinada forma, porque su comportamiento tiene sentido para ellos según la manera que tienen de experimentar el mundo como miembros de una cultura en particular. Por lo tanto, los consumidores de drogas, también escogerían ésta actividad y otras relacionadas, por lo significativas que resultan dentro de su medio, en el cual pueden ser reconocidos por ejecutarlas, otorgándoles un $\mathrm{Ro} / \mathrm{o}$ un espacio de pertenencia a un grupo.

Otro concepto de este modelo, la Competencia percibida, que se refiere a las creencias de una persona sobre sus áreas de capacidad, tales como la destreza atlética, habilidad académica y competencia social. En este sentido, vuelve a aparecer el escaso conocimiento de capacidades que el individuo consumidor de drogas tiene sobre sí mismo, restringiendo sus áreas de capacidad a aquellas que le otorgan resultados positivos o satisfactorios (5). Esto generalmente ocurre, por ejemplo en adolescentes consumidores de drogas que desertan del sistema escolar, sistema en el que no se reconocen como personas capaces y el cual abandona para dedicarse a actividades de consumo que sienten pueden realizar satisfactoriamente, entre ellas la venta y tráfico de drogas.

\section{Método}

Este es un estudio cualitativo transversal de tipo exploratorio. La selección de los participantes en el estudio se realizó en primera instancia consultando el listado de Centros de Tratamiento y Rehabilitación con Programa Ambulatorio Intensivo de la Región Metropolitana obtenido de la base de datos del CONACE. Se verificó vía telefónica la disponibilidad de Terapeutas Ocupacionales en cada institución, logrando contactar a sólo cuatro profesionales. La escasez de Terapeutas Ocupacionales trabajando en ésta área tiene relación con los hallazgos encontrados en estudios anteriores (6). Debido al bajo número de participantes contactados, se decidió ampliar el colectivo de estudio, incorporando Terapeutas Ocupacionales con experiencia en otros programas de tratamiento e incluyendo a un profesional de la $\mathrm{V}$ región. Finalmente el colectivo de estudio se constituyó con siete Terapeutas Ocupacionales. El criterio utilizado para determinar el número de entrevistas a realizar fue el de saturación de datos, esto 
es, hasta el punto en que ya no se obtiene nueva información y ésta comienza a ser redundante.

Los siete entrevistados, se encuentran ejerciendo la disciplina en Centros de Tratamiento y Rehabilitación que cuentan con convenio CONACE-MINSAL-FONASA; estos corresponden a: Comunidad Terapéutica Dianova, Comunidad Terapéutica Talita Kum, COSAM Conchalí, COSAM San Bernardo, CTR Peñablanca y Unidad de Psiquiatría Comunitaria del Hospital Padre Hurtado. Además uno de ellos se desempeña actualmente en el CONACE. El promedio de años de experiencia de los profesionales entrevistados es de 3 años 9 meses.

Tabla 1

Años de experiencia de los entrevistados

\begin{tabular}{|c|c|}
\hline Años de experiencia & Números de T.O. \\
\hline Menos de $\mathbf{1}$ año & 1 \\
\hline Entre $\mathbf{1}$ y $\mathbf{5}$ años & 5 \\
\hline Más de $\mathbf{5}$ años & 5 \\
\hline Total & 7 \\
\hline
\end{tabular}

La recolección de datos fue a través de una entrevista semiestructurada. Primero, se construyó un formato piloto de entrevista compuesta por seis preguntas con respuestas abiertas. Estas preguntas buscaban recopilar información con respecto a la etapa de ingreso a los programas de drogodependencias, aspectos evaluados desde la Terapia Ocupacional, metodología de evaluación de Terapia Ocupacional en etapa de ingreso y durante el proceso de intervención, áreas ocupacionales que se observan con mayores déficits y otros aspectos desde la perspectiva de la disciplina que serían relevantes de evaluar.

Tras la primera aplicación de ésta entrevista semiestructurada y en base al análisis de la información recopilada, se corrigieron las preguntas con el fin de asegurar que éstas recogieran de forma certera la información requerida. Finalmente la entrevista quedó constituida por cinco preguntas. Cada entrevista fue realizada por dos investigadores. La información fue registrada por medio de notas y grabación. Las entrevistas fueron transcritas en tiempo verbal, y posteriormente analizadas por el grupo de investigadores, estableciendo categorías que unieran criterios para facilitar el análisis.

Se solicitó a los siete participantes consentimiento informado para la recolección de información a través de la grabación y toma de notas, para la utilización de dicha información con fines de investigación y para la posterior publicación del estudio.

\section{Resultados}

Los resultados serán presentados de acuerdo al orden de las preguntas realizadas en la entrevista a los participantes. Ante la pregunta $N^{\circ} 1$, “¿Qué aspectos ocupacionales evalúa en la etapa de ingreso?", se encontraron 25 aspectos ocupacionales susceptibles a evaluar en etapa de ingreso por los entrevistados, que oscilan desde rutina y hábitos, historia laboral, hasta ambiente de desempeño ocupacional. Para facilitar el análisis, las respuestas obtenidas fueron clasificadas en las siguientes componentes ocupacionales, en base al criterio y marco teórico de los investigadores:

- Ambiente: que incluye las respuestas relativas a ambiente social, laboral y físico.

- Educación: que incluye las respuestas relativas a antecedentes y expectativas educacionales.

- Eventos críticos: que incluye las respuestas relativas a experiencias significativas (positivas y negativas) y logros.

- Habilidades: que incluye las respuestas relativas a habilidades de desempeño, con especial énfasis en habilidades socio-laborales y cognitivas.

- Historia Laboral: que incluye las respuestas relativas a antecedentes laborales, fortalezas y debilidades en el trabajo.

- Roles: que incluye las respuestas relativas a roles en general y en específico a rol de trabajador. 
- Rutina y hábitos: que incluye las respuestas relativas a estructuración de la rutina, equilibrio ocupacional y distribución del tiempo.

- Tiempo libre: que incluye las respuestas relativas a tiempo libre propiamente tal y ocio.

- Volición: que incluye las respuestas relativas a motivación, intereses, sentido de eficacia, valores y elecciones ocupacionales.

En base a estas categorías, las respuestas obtenidas en la pregunta $\mathrm{N}^{0} 1$ se distribuyen como muestra la tabla 2 .

Tabla 2

Distribución de los componentes ocupacionales evaluados en la etapa de ingreso

\begin{tabular}{|c|c|c|}
\hline Categoría & Razón & $\%$ \\
\hline Rutina y hábitos & $6 / 7$ & 86 \\
\hline Roles & $6 / 7$ & 86 \\
\hline Volición & $5 / 7$ & 71 \\
\hline Tiempo Libre & $5 / 7$ & 71 \\
\hline Historia Laboral & $5 / 7$ & 71 \\
\hline Habilidades & $3 / 7$ & 43 \\
\hline Educación & $3 / 7$ & 43 \\
\hline Ambiente & $3 / 7$ & 43 \\
\hline Eventos críticos & $3 / 7$ & 43 \\
\hline
\end{tabular}

Según muestra la tabla 2, son evaluadas por más del $50 \%$ de los Terapeutas Ocupacionales, los componentes ocupacionales de rutina/hábitos, roles, volición, tiempo libre e historia laboral.

Del $86 \%$ de los terapeutas ocupacionales que reportó evaluar en la etapa de ingreso, rutina y hábitos, el 14\% evalúa hábitos a través de una pauta estructurada como la Pauta de Observación de Desempeño Ocupacional creada por el centro. Otro 14\% evalúa rutina de aseo a través de la Observación Clínica durante el desempeño. El resto de los profesionales no reportaron la utilización de algún método de evaluación específico. Sin embargo, la información referida a rutina podría ser inferida desde la Entrevista para la Integración SocioOcupacional, referido a organización del tiempo, que es aplicada por el $57 \%$ del colectivo de estudio.

El $86 \%$ de los participantes reportó evaluar en la etapa de ingreso, roles, pero sólo el $43 \%$ de los participantes evalúa roles a través de una pauta estructurada como el Listado de Roles. El resto de los TO utilizan métodos de evaluación no estructurados.

El $71 \%$ de los entrevistados reportó evaluar en la etapa de ingreso, aspectos del componente volicional. De estos aspectos, sólo son evaluados los intereses a través de una pauta estructurada como es, el Listado de Intereses, que es aplicada por el $57 \%$ de los terapeutas ocupacionales entrevistados. Los otros aspectos de sentido de eficacia, valores y actitudes volitivas, son evaluados por métodos creados por cada Terapeuta, que pueden ser pautas de observación, entrevistas u observación clínica.

El $71 \%$ de los entrevistados reportó evaluar en la etapa de ingreso, las áreas de desempeño ocupacional referidas a Trabajo y Tiempo Libre, pero no reportaron la utilización de algún método de evaluación específico.

Ante la pregunta $N^{\circ} 2$, "¿Qué modelos o marcos de referencia propios de Terapia Ocupacional utiliza en la evaluación? Mencione y describa todas evaluaciones (pautas formales e informales) utilizadas según modelos o marcos de referencias", se encontró que el 100\% de los entrevistados menciona como Modelo de Terapia Ocupacional utilizado, el Modelo de Ocupación Humana, refiriendo aplicar tanto instrumentos propios de éste, como pautas de entrevista y observación elaboradas en base a él. Además, un importante número de ellos agrega a su respuesta, Modelos que no son propios de la disciplina. Esto coincide con los resultados que arrojó la investigación realizada en el año 2005 (6), donde se destaca, una heterogeneidad en los enfoques de evaluación e intervención, llevados a cabo en los Programas de Rehabilitación de Drogodependencia en la Región Metropolitana. Así, dos de los siete Terapeutas Ocupacionales entrevistados, menciona emplear el Modelo Cognitivo-Conductual. Dos de los siete, menciona emplear el Modelo Sistémico. Uno de los siete, menciona emplear el Modelo Transteórico. Uno de los siete, 
menciona emplear el Modelo de Reinserción Psicosocial y uno de los siete, menciona el Modelo Ecológico Social.

Los entrevistados identificaron los siguientes métodos de evaluación:

- Listado de roles.

- Evaluación de Habilidades de Interacción y Comunicación (ACIS).

- Listado de Intereses.

- Ficha Autoaplicada Socio-Ocupacional.

- Entrevista para la Integración Socio-Ocupacional.

- Entrevista Semiestructurada: algunas basadas en el Modelo de Ocupación Humana.

- Observación Clínica durante desempeño: incluye respuestas relativas a observación en rutinas de aseo, alimentación, talleres, actividades recreativas y grupales.

- Pautas de Observación de Desempeño Ocupacional creada por el centro: incluye habilidades, competencias, hábitos, intereses, habilidades cognitivas, hábitos laborales, relaciones interpersonales y actitudes volitivas.

Las respuestas obtenidas en esta pregunta no. 2 se distribuyen como muestra la Tabla 3:

Tabla 3

Distribución de métodos de evaluación utilizados

\begin{tabular}{|c|c|c|}
\hline Instrumentos & Razón & $\%$ \\
\hline $\begin{array}{c}\text { Ficha Autoaplicada } \\
\text { Socio-Ocupacional }\end{array}$ & $5 / 7$ & 71 \\
\hline Observación Clínica & $5 / 7$ & 71 \\
\hline Listado de Intereses & $4 / 7$ & 57 \\
\hline $\begin{array}{c}\text { Entrevista para la Integración } \\
\text { Socio-Ocupacional }\end{array}$ & $4 / 7$ & 57 \\
\hline Listado de roles & $3 / 7$ & 43 \\
\hline Entrevista Semiestructurada & $3 / 7$ & 43 \\
\hline ACIS & $1 / 7$ & 14 \\
\hline Pautas de observación & $1 / 7$ & 14 \\
\hline
\end{tabular}

Según muestra la tabla 3, los métodos de evaluación más utilizados por los Terapeutas Ocupacionales participantes, corresponden a la Ficha Autoaplicada SocioOcupacional y a la observación clínica con un 71\%; mientras que los métodos de evaluación menos utilizados corresponden al ACIS y pautas de observación creados por los centros de atención.

Ante la pregunta № 3: "En relación a la evaluación realizada, ¿Qué aspectos ocupacionales se observan con mayores déficits? Mencione los aspectos deficitarios", se encontraron 22 componentes ocupacionales deficitarios que oscilan desde trabajo y tiempo libre, hasta habilidades de comunicación e interacción, roles, rutinas y hábitos. Estos últimos alcanzan bajos porcentajes y se contradicen con los resultados de la descripción del Perfil Ocupacional del Consumidor de Drogas (5), donde se deduce un deterioro importante de los hábitos, rutinas y roles. Para facilitar el análisis, las respuestas obtenidas fueron clasificadas en las siguientes nueve categorías, en base al criterio y marco teórico de los investigadores:

\section{- Entorno social.}

- Estructuración de Rutina.

- Habilidades de comunicación e interacción: que incluye las respuestas relativas a habilidades sociales, relaciones interpersonales y habilidades de comunicación.

- Hábitos.

- Rol de madre y trabajador.

- Trabajo: que incluye las respuestas relativas a capacitación laboral y motivación al trabajo.

- Uso del Tiempo libre: que incluye las respuestas relativas a variedad de actividades de tiempo libre, experiencias y capacidad de disfrutar en las actividades.

- Volición: que incluye las respuestas relativas a motivación, intereses, causalidad personal y espiritualidad.

En base a estas categorías, las respuestas obtenidas en la pregunta número 3 se distribuyen como muestra la tabla 4. 
Tabla 4

Distribución de los componentes ocupacionales con mayor déficit

\begin{tabular}{|c|c|c|}
\hline Componente ocupacionales & Razón & $\%$ \\
\hline Trabajo & $6 / 7$ & 86 \\
\hline Uso Tiempo Libre & $6 / 7$ & 86 \\
\hline Volición & $5 / 7$ & 71 \\
\hline $\begin{array}{c}\text { Habilidades de Comunicación } \\
\text { e interacción }\end{array}$ & $3 / 7$ & 43 \\
\hline Rol de madre y trabajador & $3 / 7$ & 43 \\
\hline Entorno Social & $2 / 7$ & 29 \\
\hline Hábitos & $2 / 7$ & 29 \\
\hline Estructuración de la Rutina & $1 / 7$ & 14 \\
\hline
\end{tabular}

Según muestra la tabla 4, más del $50 \%$ de los Terapeutas Ocupacionales participantes menciona como categorías ocupacionales con mayores déficit el trabajo, uso del tiempo libre y volición.

Ante la pregunta no. 4: "Durante el proceso de intervención ¿Realiza otras evaluaciones? Si su respuesta es sí, mencione y describa cuáles", se encontraron diversas evaluaciones, que oscilan desde habilidades de desempeño, dinámica familiar hasta volición. Estos datos se exponen en la tabla número 5.

Tabla 5

Distribución otras evaluaciones realizadas durante el proceso de intervención

\begin{tabular}{|c|c|c|}
\hline Componente ocupacional evaluado & Razón & $\%$ \\
\hline Habilidades de Desempeño & $5 / 7$ & 71 \\
\hline Rutina Diaria & $2 / 7$ & 29 \\
\hline Entorno & $2 / 7$ & 29 \\
\hline Dinámica Familiar & $2 / 7$ & 29 \\
\hline Puesto de Trabajo & $1 / 7$ & 14 \\
\hline Pauta del Mediador & $1 / 7$ & 14 \\
\hline Historia Laboral & $1 / 7$ & 14 \\
\hline
\end{tabular}

\begin{tabular}{|c|c|c|}
\hline Tiempo Libre & $1 / 7$ & 14 \\
\hline Productividad & $1 / 7$ & 14 \\
\hline Espiritualidad & $1 / 7$ & 14 \\
\hline Volición & $1 / 7$ & 14 \\
\hline
\end{tabular}

Según muestra la tabla 5, que la mayoría de los entrevistados (71\%) coincide en evaluar habilidades de desempeño durante el proceso de intervención; sin embargo, en el resto de aspectos referidos, existe escasa concomitancia entre los profesionales.

Ante la última pregunta no. 5, "De acuerdo a su experiencia, ¿Existen otros aspectos, desde la perspectiva ocupacional, que consideraría relevantes de evaluar? Si la respuesta es sí, mencione cuáles", se encontraron una diversidad de respuestas, que oscilan desde la necesidad de evaluar capacitación y habilidades de desempeño laboral, hasta la inexistencia de aspectos adicionales a evaluar. De acuerdo a estos datos, se construyen 4 categorías, en base al criterio y marco teórico de los investigadores, que se exponen en la siguiente tabla:

Tabla 6

Distribución otros componentes relevantes a evaluar

\begin{tabular}{|c|c|c|}
\hline Otros componentes a evaluar & Razón & \% \\
\hline Habilidades de desempeño laboral & $2 / 7$ & 29 \\
\hline Vocación / Capacitación laboral & $2 / 7$ & 29 \\
\hline No refiere otros aspectos & $2 / 7$ & 29 \\
\hline Entorno Espiritual & $1 / 7$ & 14 \\
\hline
\end{tabular}

Según muestra la tabla 6 más del 50\% de los profesionales participantes consideran que existen otros componentes ocupacionales relevantes a evaluar. Dentro de ellos, destaca la necesidad de evaluar aspectos referidos al área del trabajo, la mitad reportó que deben evaluarse habilidades de desempeño laboral; mientras que la otra mitad, reportó que deben evaluarse vocación y/o capacitación laboral. Este último refiere la necesidad de contar con una pauta estandarizada, que permitiese proyectar el nivel de integración laboral al cual puede acceder una persona por medio del desempeño de un rol productivo. 
Por otro lado, el $100 \%$ de los entrevistados refiere la necesidad de contar con pautas de evaluación acotadas, de fácil y corta aplicación, debido a que la relación entre la población beneficiaria y las horas que disponen los profesionales de la disciplina, no son suficientes para realizar evaluaciones extensas como son las propuestas por el Modelo de Ocupación Humana.

\section{DısCUSIÓN}

Este estudio reafirma los resultados encontrados en estudios anteriores $(6,7)$ que hacen referencia a una carencia de homogeneidad entre los modelos utilizados por Terapeutas Ocupacionales en el área del Consumo Problemático de Drogas. Además, este estudio revela un uso importante de modelos no propios de la disciplina. El modelo común por todos los entrevistados, es el Modelo de Ocupación Humana. Sin embargo, la similitud de conceptos empleados por los profesionales, responde al conocimiento generalizado de este modelo. Por lo tanto, el uso de este modelo no asegura un correcto uso de su terminología, al existir distintas interpretaciones de éstos en cada Centro de Tratamiento y Rehabilitación.

Sumado a lo anterior, al existir una gran variabilidad en la terminología utilizada para hacer referencia a los aspectos ocupacionales, es entorpecido el entendimiento entre distintos Terapeutas Ocupacionales que trabajan en la misma área. Por ejemplo, para hacer referencia a la rutina, los entrevistados emplean diversos términos como equilibrio ocupacional, repartición y estructuración del tiempo. Por lo tanto este estudio, da cuenta de la necesidad de aunar criterios y poseer un lenguaje común entre los exponentes de la disciplina, ya que esto facilita la comunicación, el intercambio de experiencias y también permite elaborar registros e investigaciones forjados en una misma base o sustento conceptual.

Para dar respuesta a ésta necesidad, se propone el amplio conocimiento y uso del Marco de Trabajo para la práctica de la Terapia Ocupacional (11), creado por la Asociación Americana de Terapia Ocupacional (AOTA), que establece una base y terminología común para estos profesionales, con el fin de favorecer el intercambio de información y potenciar la labor, en beneficio de los usuarios.

Por otro lado, esta investigación reveló una amplia variabilidad en los aspectos ocupacionales a evaluar, con una diversidad aún mayor de métodos de valoración. En general, estos corresponden a evaluaciones no estructuradas, ni estandarizadas, lo que también es perjudicial para el entendimiento dentro de la disciplina, limitando el proceso de desarrollo y perfeccionamiento del trabajo en esta área. Al existir un amplio número de elementos a valorar, se condiciona un periodo extenso de evaluación, que complejiza el inicio de la intervención y la focalización de ésta. Por ello, es importante contar con métodos que permiten obtener la mayor cantidad de información en el menor tiempo posible, que a la vez sean simples y respondan a la relación costo-efectividad. De esta manera, se podría optimizar la labor del Terapeuta Ocupacional utilizando el recurso técnico disponible en los distintos centros. Esto quiere decir, que al poseer herramientas simples de evaluación, éstas pueden ser aplicadas por personal técnico, previamente capacitado en la recopilación de la información; tomando en consideración que ellos cuentan con una mayor cantidad de horas asignadas para el trabajo en salud. Cabe destacar que la información recogida, tendría que ser indudablemente analizada con posterioridad por el Terapeuta Ocupacional, quien establecerá el diagnóstico ocupacional, determinando las necesidades y el plan a seguir con cada usuario.

Los instrumentos simplificados de evaluación de Terapia Ocupacional pueden ser aplicados por los distintos profesionales y/o técnicos en el área de drogodependencias, pero el Terapeuta Ocupacional es el profesional indicado para realizar un análisis ocupacional que guíe la intervención de la disciplina. Resulta necesario capacitar a los distintos entrevistadores en la aplicación de estos instrumentos con el fin de profundizar y rescatar aquellos aspectos más importantes para un análisis ocupacional adecuado. De aquí también se 
desprende la necesidad de realizar nuevas investigaciones en el área, pudiendo ser tema para próximos trabajos de investigación, la determinación o elaboración de dichos instrumentos.

Según los hallazgos encontrados en esta investigación, los componentes ocupacionales prioritarios a evaluar en etapa de ingreso son rutina, hábitos, roles, volición, tiempo libre e historia laboral. La evaluación de ingreso entrega información de base que es necesario complementar con las evaluaciones realizadas durante el proceso de tratamiento, con el fin de lograr un análisis ocupacional integral. Las habilidades y capacitaciones laborales corresponden a aspectos ocupacionales posibles y relevantes de evaluar, por sobre el 50\% de los Terapeutas Ocupacionales; pero no constituyen parte específica de una evaluación inicial y durante el proceso de intervención.

En relación a esto, es posible concluir que el perfil de habilidades laborales constituyen aspectos fundamentales a valorar desde Terapia Ocupacional en el área del Consumo Problemático de drogas, lo cual se complementa con la línea de intervención que el CONACE establece actualmente, que potencia la reinserción Socio-laboral del usuario. De acuerdo al cúmulo de necesidades pesquisadas en esta área, se abre una invitación a la investigación en ésta área, con el fin de generar evidencia que facilite el trabajo del Terapeuta Ocupacional y por sobre todo, que favorezca el estado de salud e integración social de las personas con consumo problemático de drogas.

\section{ReferenCias Bibliograficas}

(1) Consejo Nacional para el Control de Estupefacientes. "Programa para la Integración Socio Ocupacional de personas drogodependientes; Abriendo Puertas" 2004, República de Chile. Ministerio del Interior. Disponible en internet: http://www.bibliodrogas. cl/biblioteca/digital/TRATAMIENTO_CL_5132.PDF (consultado el 12 de Diciembre de 2009).
(2) Consejo Nacional para el Control de Estupefacientes. "Octavo estudio nacional de drogas en población general de Chile" 2008, República de Chile. Ministerio del Interior. Disponible en internet: http://bibliodrogas.cl/biblioteca/digital/resumen_informe_VIII_estudio_drogas_poblaciongeneral_junio2009.pdf (consultado el 10 de Enero de 2010).

(3) Consejo Nacional para el Control de Estupefacientes. "Séptimo estudio nacional de drogas en población escolar de Chile" 2007, República de Chile. Ministerio del Interior. Disponible en internet: http://bibliodrogas.cl/biblioteca/digital/ vii\%20estudio/informe_ejecutivo_Estudio_Escolares_Conace2007_16-06-2008.pdf (consultado el 11 de Enero de 2010).

(4) Consejo Nacional para el Control de Estupefacientes. "Séptimo estudio nacional de drogas en población general de Chile" 2006, República de Chile. Ministerio del Interior. Disponible en internet: http://bibliodrogas.cl/biblioteca/digital/ vii\%20estudio/Introduccion.pdf (consultado el 10 de Enero de 2010)

(5) RIVEROS M.“Historia de desempeño ocupacional en usuarios de programas de tratamiento y rehabilitación en drogas: recomendaciones para una intervención". Tesis para optar al grado de Magíster en Salud Pública. Estudio complementario a la investigación "Proyecto de asesoría al Consejo Nacional de Control de Estupefacientes - CONACE" 2004, Escuela de Salud Pública, Universidad de Chile.

(6) AVILÉS S, BANDA R, FIGUEROA M. Seminario "Terapia Ocupacional en Drogodependencia: Relevancia del uso de la perspectiva de la Ocupación para la intervención" 2005. Escuela de Terapia Ocupacional, Universidad de Chile.

(7) AGUILERA M, JIMÉNEZ S, MIRANDA A, PIZARRO C. Seminario "Desempeño ocupacional disfuncional en personas con consumo problemático de drogas" 2006. Escuela de Terapia Ocupacional, Universidad de Chile. 
(8) AGUIRRE R, RIVEROS M. "Intervención de terapia ocupacional en programas de tratamiento y rehabilitación en drogas". Revista Chilena de Terapia Ocupacional 2002; 2: 35-39. Disponible en internet: http://www.revistas.uchile.cl/index.php/RTO/ article/viewArticle/138/122 (consultado 19 de Mayo de 2010).

(9) MUÑOZ I, RIVEROS M. Trabajo"Rediseño de Ocupaciones en Jóvenes con Consumo Problemático de Drogas" 2006. Trabajo para aprobar Curso: Introducción a la Ciencia de la Ocupación. Escuela de Terapia Ocupacional, Universidad de Chile.

(10) YERXA E. "Health and the human spirit for occupation". Am J Occup Ther. 1998; 56 (6): 412-418.

(11) American Occupational Therapy Association. "Occupational therapy practice framework: Domain and process". Am J Occup Ther. 2002; 56 (6): 609639.
(12) Naciones Unidas. "Manual Abuso de drogas: Tratamiento y Rehabilitación. Guía práctica de planificación y Aplicación" 2003. Capítulo II: Definición de tratamiento. Oficina contra la droga y el delito. Disponible en internet: http://www.unodc.org/pdf/report_2003-07-17_1_es.pdf (consultado 08 de Febrero de 2010)

(13) American Psychiatric Association. "Diagnostic and Statistical Manual of Mental Disorders"4th $\mathrm{ed}$. 1994, Washington, D.C.: Author.

(14) KIELHOFNER G. "Modelo Ocupación Humana: Teoría y Aplicación. Instrumentos y Evaluación". $3^{\text {rd }}$ ed. 2004. Capítulos 13-17. 